\title{
Avaliação da medida do perímetro braquial como metodologia de triagem de crianças pré-escolares obesas
}

\author{
Assessment of mid-upper arm circumference as a method \\ for obesity screening in preschool children

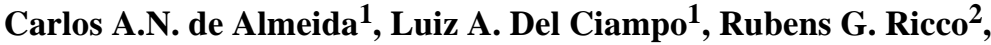 \\ Sergio M. Silva Jr. ${ }^{3}$, Rosiane B. Naves ${ }^{3}$, Jennifer F. Pina ${ }^{3}$
}

\section{Resumo}

Objetivo: O presente estudo visou a avaliar se o perímetro braquial poderia ser utilizado para a triagem de crianças obesas na idade pré-escolar.

Método: 1.090 crianças com idades entre 12 e 59,99 meses foram estudadas, obtendo-se informações pessoais, além de peso, estatura e perímetro braquial. Foram obtidas as sensibilidades e especificidades de diferentes escores $\mathrm{z}$ de perímetro braquial para idade e perímetro braquial para estatura, para a detecção de crianças obesas, definidas como aquelas que apresentaram escore $\mathrm{z}$ de peso para estatura acima de 2 .

Resultados: Das 1.090 crianças avaliadas, 6,6\% apresentaram escore $\mathrm{z}$ de peso para estatura acima de 2 . O escore $\mathrm{z}$ de 0,7 do indicador perímetro braquial para idade determina a melhor associação entre sensibilidade $(76,5 \%)$ e especificidade $(77,9 \%)$, para a detecção das crianças obesas. $\mathrm{O}$ escore $\mathrm{z}$ de 0,6 do indicador perímetro braquial para estatura determina a melhor associação entre sensibilidade $(79,4 \%)$ e especificidade $(77,6 \%)$ para a detecção das crianças obesas.

Conclusões: Não se observou vantagem do uso do perímetro braquial relacionado à estatura quando comparado à sua relação com a idade. Dessa forma, propõem-se pontos de corte, utilizando-se apenas o indicador perímetro braquial para idade que, pela sua facilidade e rapidez de obtenção em populações, é um método alternativo viável para triagem de crianças pré-escolares portadoras de obesidade.

J Pediatr (Rio J) 2003;79(5):455-60: Obesidade, perímetro braquial, crianças, antropometria, avaliação nutricional.

\section{Introdução}

A prevalência de obesidade no Brasil vem aumentando em todas as faixas etárias. Especificamente entre as crian-

1. Doutor em Pediatria pela USP.

2. Livre Docente em Pediatria pela USP

3. Acadêmico de Medicina da Universidade de Ribeirão Preto.

Artigo submetido em 27.02.03, aceito em 11.06.03.

\section{Abstract}

Objectives: To evaluate if mid-upper arm circumference (MUAC) can be used for obesity screening in preschool children.

Methods: 1,090 children aged from 12 to 59.99 months were studied. Personal information, weight, height and MUAC were obtained. Sensitivity and specificity to detect obese children were calculated for MUAC to age and MUAC to height z scores. Obesity was defined as weight to height $\mathrm{z}$ score $>2$.

Results: $6.6 \%$ of the children had a weight to height $\mathrm{z}$ score $>2$. The best association between sensitivity (76.5\%) and specificity (77.9\%) to detect obese children for MUAC to age was obtained with a $\mathrm{z}$ score of 0.7 . In terms of MUAC to height, the best association between sensitivity $(79.4 \%)$ and specificity $(77.6 \%)$ to detect obese children was obtained with a 0.6 score.

Conclusions: MUAC to height $\mathrm{z}$ score was not advantageous for obesity screening when compared to MUAC to age z score. MUAC to age $\mathrm{z}$ score seems to be an adequate alternative method for obesity screening in preschool children.

J Pediatr (Rio J) 2003;79(5):455-60: Obesity, mid upper arm circumference, children, anthropometry, nutritional assessment.

ças pré-escolares, passou de 2,1\%, em $1989^{1}$, para 4,1\% em $1996^{2}$. Esse aumento segue a tendência da transição nutricional vivenciada especialmente naquelas regiões do mundo que atravessam períodos de desenvolvimento socioeconômico. Dessa forma, é bastante provável que a epidemia de obesidade atualmente vivida pelos Estados Unidos da América, onde a prevalência entre crianças menores de 
cinco anos era de 10,3\% em $1997^{3}$, também aconteça entre as crianças brasileiras, à medida que o país se desenvolve.

Uma das principais estratégias de combate à obesidade está em sua prevenção e detecção precoce, permitindo que se consigam mais facilmente propor e implementar as mudanças no comportamento alimentar indispensáveis ao seu tratamento. Essa necessidade esbarra, entretanto, na dificuldade diagnóstica decorrente de diversos fatores, como a relativa boa aceitação cultural da criança obesa, o despreparo dos profissionais, muito mais treinados para detectar e tratar a subnutrição, e a metodologia de avaliação populacional, fundamentada basicamente na obtenção e análise de duas medidas antropométricas, peso e estatura. Dessa forma, seria de grande utilidade o desenvolvimento de estratégias de triagem universal, baseadas em metodologia simples, barata e eficaz, realizadas nos locais de permanência da criança, como creches e escolas, a fim de que o pediatra, ao receber e avaliar a criança previamente triada, já estivesse atento ao problema e encontrasse facilidade no estabelecimento do diagnóstico e da terapêutica. Diante desses princípios, uma possível alternativa para esse fim seria a utilização da medida do perímetro braquial. Em 1997, pesquisadores da OMS publicaram novas tabelas com valores de escores $\mathrm{z}$ de perímetro braquial para idade e para estatura, recomendando seu uso para abordagem da subnutrição $0^{4,5}$. O presente estudo visou avaliar se esse indicador poderia também ser utilizado na avaliação da obesidade, especialmente como método de triagem populacional, selecionando as crianças em maior risco de obesidade, as quais seriam, posteriormente, avaliadas através de outros métodos mais complexos.

\section{Material e método}

No início do ano de 1999, todas as creches e pré-escolas oficialmente reconhecidas pela Secretaria da Educação da cidade de Ribeirão Preto foram identificadas, encontrandose nelas matriculadas 24.706 crianças em idade pré-escolar, sendo 12.906 na rede pública e 11.800 na rede privada. Após contato com os responsáveis, 30 instituições aceitaram participar do estudo, sendo 23 públicas e 7 privadas, perfazendo 2.063 crianças, das quais $1.090 \mathrm{com}$ idades entre 12 e 59,99 meses.

Após a obtenção de autorização escrita dos responsáveis, conforme norma nacional para pesquisas envolvendo seres humanos ${ }^{6}$, e de aprovação pelo comitê de ética médica da Universidade de Ribeirão Preto, de cada criança foram obtidos, em uma única ocasião, informações pessoais, peso, estatura e perímetro braquial, por uma equipe de 25 acadêmicos de medicina. Os acadêmicos foram treinados para a realização da antropometria em grupos de cinco, e foram considerados adequadamente padronizados quando a diferença entre os resultados obtidos pelos diferentes medidores era inferior a $0,5 \%$. A técnica para a obtenção das medidas seguiu recomendações internacionais ${ }^{7,8}$. O material utilizado para obtenção das medidas foi adquirido especificamente para o estudo e constou de fita métrica de metal Lufking ${ }^{\circledR}$ com escala de $1 \mathrm{~mm}$, balança eletrônica pediátrica Filizola ${ }^{\circledR}$ com capacidade máxima de $16 \mathrm{~kg}$ e escala de $10 \mathrm{~g}$ (utilizada em todas as crianças que não se mantinham em pé sem apoio), balança eletrônica portátil Filizola $^{\circledR}$ com escala de $100 \mathrm{~g}$ (utilizada em crianças com mais de $15 \mathrm{~kg}$ ), antropômetro horizontal em madeira e fórmica com fita métrica em metal de $102 \mathrm{~cm}$ e escala de 5 $\mathrm{mm}$ (para medição do comprimento de crianças até dois anos de idade), e antropômetro vertical desmontável, em madeira e fórmica com fita métrica em metal de $200 \mathrm{~cm}$ e escala de $1 \mathrm{~cm}$ (para medição da estatura de crianças com mais de dois anos de idade), ambos fabricado em oficina de precisão sob orientação dos autores.

Foram obtidos os escores z dos indicadores peso para estatura, perímetro braquial para idade e perímetro braquial para estatura através do software Epi-Info $2000^{9}$, segundo os padrões do National Center for Health Statistics (NCHS $)^{10}$ e da Organização Mundial de Saúde ${ }^{4,5}$. Considerou-se como padrão-ouro para detecção de obesidade a obtenção de escore z maior que 2 para o indicador peso para estatura, seguindo recomendação da Organização Mundial da Saúde ${ }^{1}$. Em seguida, foi calculada a sensibilidade e a especificidade de diferentes pontos de corte de escore $\mathrm{z}$ dos indicadores perímetro braquial para idade e perímetro braquial para estatura, para detecção da obesidade, partindo-se do valor que levava à sensibilidade de $100 \%$ até aquele que fornecia especificidade de $100 \%$, utilizando-se intervalos de 0,1 escore z. Dessa forma, para o indicador perímetro braquial para idade, o intervalo de avaliação foi de $-0,9$ a 3,2 e, para o indicador perímetro braquial para estatura, de -0,7 a 3,5. Após a obtenção do melhor ponto de corte, foi utilizado o programa Epi-Info $2000^{9}$ para se obter os valores de perímetro braquial correspondentes a esse ponto para meninos e meninas, de modo a se criar um gráfico de uso prático para a triagem populacional da obesidade em campo.

\section{Resultados}

Das 1.090 crianças avaliadas, 68 apresentaram escore z de peso para estatura acima de 2 , levando à prevalência de obesidade de $6,6 \%$.

A Figura 1 mostra a evolução dos valores de sensibilidade e especificidade dos escores $\mathrm{z}$ do indicador perímetro braquial para idade, para detecção da obesidade. Observase que o escore $z$ de 0,7 determina a melhor associação entre sensibilidade e especificidade, com valores de, respectivamente, $76,5 \%$ e $77,9 \%$. Nesse ponto, o método seleciona 278 crianças do total de 1.090 . Das 68 obesas, o método teria triado corretamente $52(76,5 \%)$, mas, para isso, teria incluído incorretamente mais 226 crianças. Por outro lado, das 812 crianças não selecionadas, restariam ainda 16 $(23,5 \%)$ obesas que não teriam sido triadas.

A Figura 2 mostra a evolução dos valores de sensibilidade e especificidade dos escores $\mathrm{z}$ do indicador perímetro 


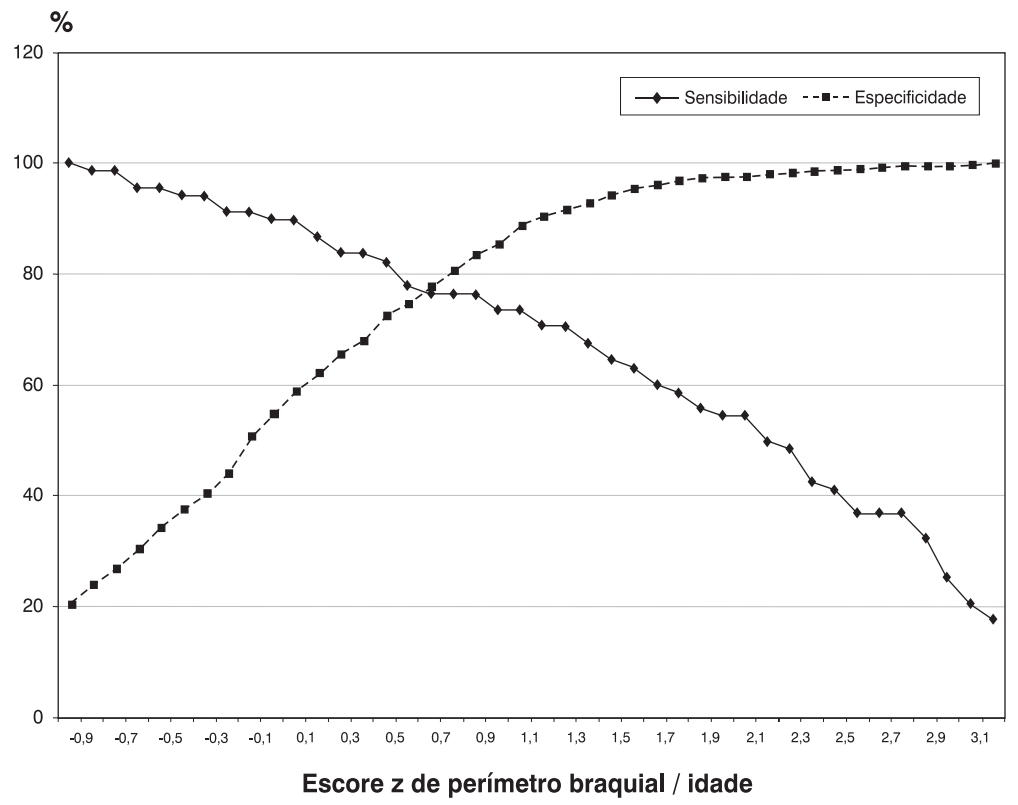

Figura 1 - Sensibilidade e especificidade de diferentes pontos de corte de perímetro braquial para idade, para a detecção de obesidade em crianças pré-escolares

braquial para estatura, para detecção da obesidade. Observa-se que o escore $\mathrm{z}$ de 0,6 determina a melhor associação entre sensibilidade e especificidade, com valores de, respectivamente, $79,4 \%$ e $77,6 \%$. Nesse ponto, o método seleciona 283 crianças do total de 1.090. Das 68 obesas, o método teria triado corretamente $54(79,4 \%)$ mas, para isso, teria incluído, incorretamente, mais 229 crianças. Por outro lado, das 807 crianças não selecionadas, restariam ainda 14 $(20,6 \%)$ obesas que não teriam sido triadas.

Considerando-se que o objetivo do estudo foi obter uma metodologia de triagem populacional de obesidade, seria desejável que fossem escolhidos pontos de corte que levassem à alta sensibilidade. Nesse caso, considerando-se, por exemplo, uma sensibilidade de $90 \%$, das 68 crianças obesas, $61(89,7 \%)$ seriam detectadas com o indicador perímetro braquial para idade e $63(92,6 \%)$ pelo indicador perímetro braquial para estatura. Por outro lado, no primeiro caso, seriam incluídas também 462 crianças não obesas e, no segundo, 436. Esse grande número de falso-positivos tornaria a triagem bastante ineficiente, à medida que implicaria na reavaliação posterior de um grande número de crianças. Dessa forma, optou-se por considerar como melhor ponto de corte aquele que associava a melhor sensibilidade e especificidade. Como os resultados foram bastante semelhantes em relação aos dois indicadores testados, não se observou vantagem na utilização do perímetro braquial associado à estatura em relação à sua associação à idade, especialmente considerando-se a maior dificuldade operacional do primeiro, em que se necessita obter a estatura, sobre o segundo, em que é suficiente o conhecimento da idade. A Figura 3 mostra os valores da medida do perímetro braquial, obtidos a partir de dados da população de referência $^{4,5}$, correspondentes ao escore $\mathrm{z}$ de 0,7 para a faixa etária de 12 a 59,9 meses, para meninos e meninas.

\section{Discussão}

O objetivo do presente estudo foi verificar se, a exemplo da desnutrição, em que a medida do perímetro braquial é um bom indicador do estado nutricional ${ }^{11,12}$, também no caso da obesidade esse instrumento poderia ser utilizado, especialmente para a realização de triagem populacional, considerando-se que essa medida tem boa correlação com a massa corporal ${ }^{11}$. Os dados apresentados mostram que, para valores elevados de sensibilidade, ideais para essa finalidade, obtiveram-se especificidades muito baixas, levando a grande número de falso-positivos, tanto para o indicador perímetro braquial para idade quanto para o indicador perímetro braquial para estatura. Esses resultados, a princípio, sugerem que esses indicadores podem não ser adequados para esse fim. A repetição desse estudo em populações com prevalências mais elevadas de obesidade 


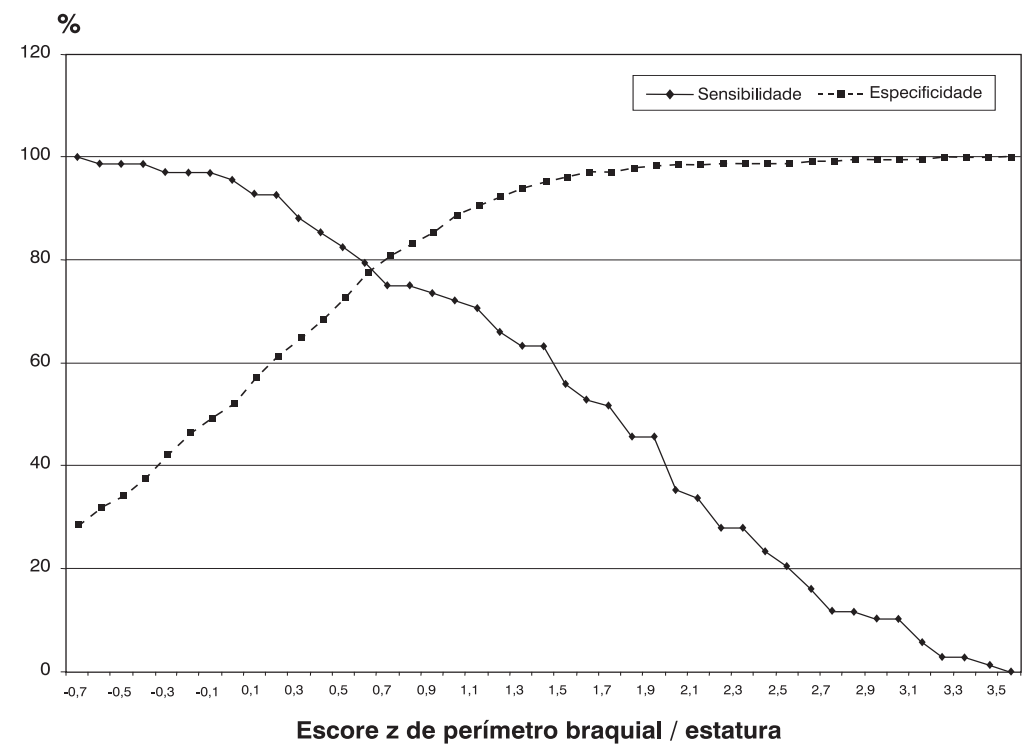

Figura 2 - Sensibilidade e especificidade de diferentes pontos de corte de perímetro braquial para estatura, para a detecção de obesidade em crianças pré-escolares

pode, eventualmente, levar a resultados distintos. Entretanto, no único trabalho semelhante ao presente estudo encontrado nas bases de dados pesquisadas, Sardinha e colaboradores, estudando adolescentes, também observaram número elevado de falso-positivos quando buscaram utilizar o perímetro braquial como método de triagem nessa faixa etária, demostrando também que uma importante limitação do método está no fato de que, para se obter valores mais altos de sensibilidade, aumenta-se o número de falsopositivos ${ }^{13}$.

Observando-se as Figuras 1 e 2, especialmente nos pontos em que se associam a melhor sensibilidade e especificidade, verificam-se resultados muito semelhantes. Esse fato permite que, para a finalidade proposta, possam ser usados indiferentemente o perímetro braquial para idade ou para estatura, mas, pela facilidade de uso, logicamente o perímetro braquial para idade deve ser preferido.

Apesar de o estudo não ter demonstrado uma eficácia ótima para o método, pode-se considerar que, frente à ausência de outras formas melhores de triagem, ele possa ser utilizado, especialmente em creches e escolas, tanto públicas como privadas, que não possuam balanças e antropômetros e tampouco pessoal preparado para seu uso e interpretação. Nesse caso, é fundamental que a técnica de medida, aparentemente simples, seja rigorosamente seguida. Observando-se a Figura 3, pode-se verificar que as diferenças entre os valores de perímetro braquial para cada idade são bastante discretas, fazendo com que pequenos erros de medida possam levar a interpretações equivocadas. Fatores como a escolha do braço adequado, a correta colocação da fita no ponto médio entre o acrômio e o olecrano, a horizontalidade do posicionamento e a força de compressão devem ser sempre levados em conta quando se trabalha com o perímetro braquial, impondo-se a rigorosa padronização dos medidores ${ }^{14}$, que não é difícil de ser obtida, demandando apenas pequeno e objetivo treinamento, uma vez que se trata de medida única, simples e com concurso de instrumental portátil, de fácil manuseio e que dispensa calibração constante. Deve-se lembrar que, no presente estudo, a medida da prega cutânea não foi avaliada como alternativa de triagem populacional de obesidade devido à dificuldade para a correta obtenção da medida, associada ao elevado custo do adipômetro.

Consideradas as limitações acima descritas, demonstrou-se, no presente estudo, que é possível realizar a triagem populacional de obesidade em crianças pré-escolares, utilizando-se apenas a idade e uma medida antropométrica facilmente realizável e de baixo custo, conseguindo-se a detecção de 76,5\% das crianças obesas, incluindo-se um número tolerável de falso-positivos que, por sua vez, não deixariam de ser beneficiados por programas educativos e preventivos que visassem à população à qual pertencem. A 


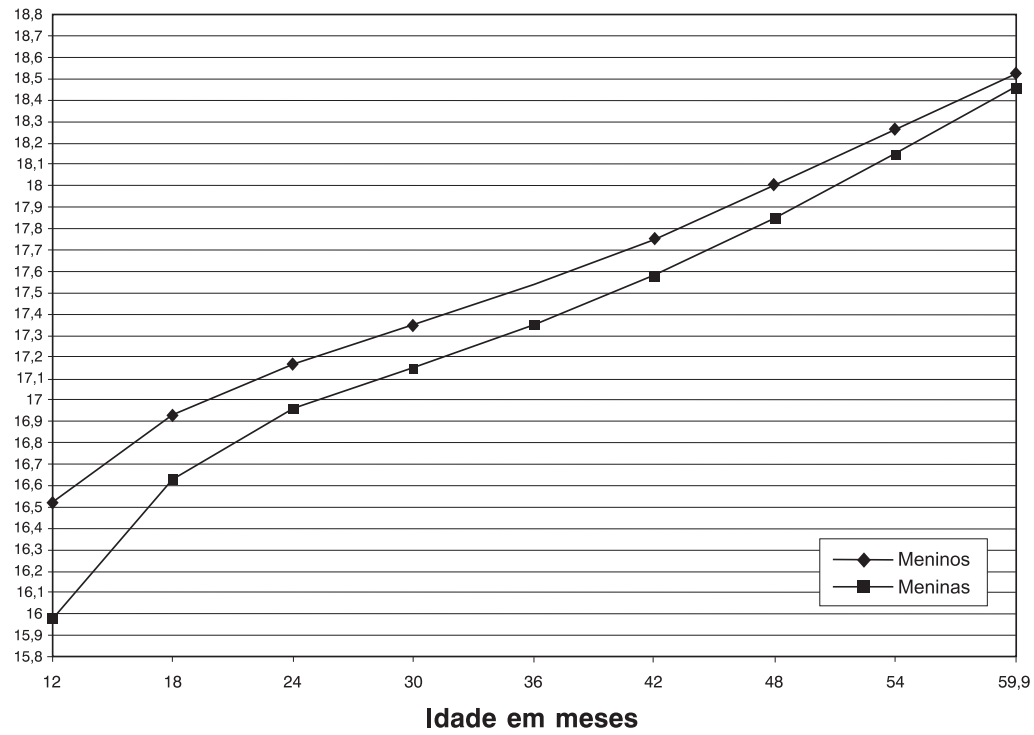

Figura 3 - Valores de perímetro braquial correspondentes ao escore $\mathrm{z}$ de 0,7 para meninos e meninas com idades entre 12 e 59,9 meses

porcentagem de falso-negativos observada $(23,5 \%)$ deve ser considerada como a principal limitação do método, já que implica em um número relevante de crianças que seriam incorretamente consideradas como não obesas. Por outro lado, para corrigir esse problema, seria necessário escolher um ponto de corte que levasse à maior especificidade, perdendo-se em sensibilidade, o que seria não condizente com um método de triagem. Assim, propõem-se os pontos de corte apresentados na Figura 3, para serem utilizados em populações que freqüentam creches e escolas do Brasil que atendam pré-escolares, como um passo inicial para se conseguir a detecção da obesidade ainda nessa faixa etária, permitindo a instituição precoce das medidas educacionais e terapêuticas, além da prevenção das comorbidades mais freqüentes entre adolescentes e adultos obesos.

\section{Referências bibliográficas}

1. WHO. Obesity: preventing and managing the global epidemic. Technical Report Series 894. Geneva; 2000.

2. Martorell R, Khan LK, Hughes ML, Grummer-Strawn LM. Obesity in Latin American woman and children. J Nutr 1998;128:1464-73.

3. Pediatric nutrition surveillance, 1997 full report. Atlanta: USA
Department of Health and Human Services, Centers for Disease Control and Prevention; 1998.

4. Onis M, Yip R, Mei Z. The development of PMB-for-age reference data recommended by a WHO Expert Committee. Bull WHO 1997;75:11-8.

5. Mei Z, Grummer-Strawn LM, Onis M, Yip R. The development of a PMB-for-height reference, including a comparison to other nutritional status screening indicators. Bull WHO 1997;75:333-41.

6. Brasil. Ministério da Saúde. Diretrizes e normas regulamentadoras de pesquisas envolvendo Seres Humanos. Brasília: Ministério da Saúde; 1997. p. 20.

7. Cameron N. The measurement of human growth. Londres: CroomHelm; 1984.

8. WHO Working Group on Infant Growth. An evaluation of infant growth: the use and interpretation of anthropometry in infants. Bull Who 1995;73(2):165-74.

9. Epi Info 2000. Programa de computador. Versão 2000. Atlanta (GE): CDC; 2000.

10. Kuczmarski RJ, Ogden CL, Guo SS, Grummer-Strawn LM, Flegal KM, Mei Z, et al. CDC growth charts: United States. Advance data from vital and health statistics; no. 314. Hyattsville, Maryland: National Center for Health Statistics; 2000.

11. Jelliffe DB, Jellliffe EF. The arm circumference as a public health index of protein-calorie malnutrition of early childhood: (XX) - current conclusions. J Trop Pediatr 1969;15:253-60.

12. De Almeida CA, Ricco RG, Del Ciampo LA, Nogueira MPC, Muccillo G. Comparison of four anthropometric methods of nutritional assessment and evaluation of the agreement between two reference populations. J Trop Pediatr 1999;45:345-50. 
13. Sardinha LB, Going SB, Teixeira PJ, Lohman TG. Receiver operating characteristic analysis of body mass index, triceps skinfold and arm girth for obesity screening in children and adolescents. Am J Clin Nutr 1999;70:1090-5.

14. Monteiro CA. Critérios antropométricos no diagnóstico da desnutrição em programas de assistência à criança. Rev Saude Publica 1984;18:209-17.
Endereço para correspondência:

Dr. Carlos Alberto Nogueira de Almeida

Rua Tamoios, 262 - ap. 154

CEP 14020-700 - Ribeirão Preto - SP

Tel./Fax: (16) 621.8130

E-mail: carlosnogueira@ directnet.com.br 